Jpn. J. Med. Sci. Biol., 51, Suppl. 1, S 124 S 128, 1998

\title{
Strategy to Prevent the Progression of Enterohemorrhagic Escherichia coli 0157 Infection to Hemolytic Uremic Syndrome
}

Tae Takeda

Department of Infectious Diseases Research, National Children's Medical Research Center, Setagaya-ku, Tokyo, Japan

\section{INTRODUCTION}

There has been a dramatic increase in the incidence of EHEC O157: H7 infections in Japan since 1996. Infections with this organism are closely associated with hemolytic uremic syndrome (HUS) due to the production of Shiga toxin (Stx) as it known by the new nomenclature. Infections with EHEC 0157:H7 have been associated with both sporadic cases and outbreaks of HUS.

Several risk factors and predictive factors for progression of the 0157 infection into HUS have been proposed such as 1) young or old age, 2) high level of leucocytosis, 3) high fever, 4) crampy abdominal pain, 5) nausea, and 6) copious bloody stool. We think all of these should be considered important. Furthermore, we want to add 7) hematuria, and 8) delayed diagnosis as risk factors (Table 1). Delayed diagnosis is one of the chief risk factor because delayed diagnosis means delayed administration of appropriate prevention strategy and also means that the disease has progressed to a stage where it may be difficult to revert. The basic approach to prevent serious illness caused by toxigenic enteropathogens would be to rapidly eliminate the etiologic agent and thereby prevent toxin accumulation in the host gut. However, antibiotic treatment of patients infected with EHEC O157:H7 has been documented to be a risk factor for the progression of enteritis to HUS 1,2).

To address the issue of the role of antibiotic use, we conducted a retrospective nation-wide survey of EHEC infection diagnosed in 1996 by questionnaires sent to 3908 hospitals all over Japan. In 1996 we had a deluge of EHEC infections 3, 4). Chief physicians from 1682 hospitals and returned the questionnaires through April 30, 1997 (response rate was 
Table 1. Risk or predictive factors for HUS

- Young or old age

- Leukocytosis

- Fever

- Abdominal pain

- Nausea

- Hematuria

- Delayed diagnosis

Table 2. Criteria to define a case of HUS

- Hemolytic anemia: Hemoglobin $<10 \mathrm{~g} / \mathrm{d} l$

- Thrombocytopenia: Platelet $<10 \times 10^{4} / \mathrm{mm}^{3}$

- Renal failure:

Oliguria/Anuria or

Creatinine $>1.5 \times$ Normal level of age

(by Japanese Society for Pediatric Nephrology)

Table 3. Retrospective Nationwide survey of STEC infection in 1996

No. of Hospitals Asked: 3908

Response Rate: 1682 (43.04\%)

No. of Patients Reported: 1271

Outbreak: 725 (57.0\%)

Sporadic: $372(29.3 \%)$

No. of all reported HUS: 199 (15.7\%)

No. of complete HUS: 107 ( $8.4 \%)$

No. of death: $5(0.39 \%)$

43.04\%). At that time the criteria for diagnosis of HUS was not properly standardized all over Japan and therefore some cases defined as HUS in this study actually were not typical HUS in terms of the symptoms described in Table 2. Counted as reported from this survey, information about 1271 cases was collected for analysis. Among these cases, the disease had progressed to HUS in $15.7 \%$ (199 cases) and there were 5 deaths. We sent second questionnaires to physicians to get more precise clinical data on these 199 HUS cases. Total number of 107 complete HUS was confirmed (Table 3). We analyzed the incidence of HUS in relation to antibiotic usage among the 1271 cases. More than $90 \%$ of the patients 
Table 4. Incidence of HUS in relation to antibiotic usage among children ( $\leqq 15$ years of age) with bacteriologically or serologically proven E. coli 0157 infection during outbreaks $^{a}$

\begin{tabular}{lcccccc}
\hline $\begin{array}{l}\text { Antibiotic } \\
\text { administration }\end{array}$ & $\begin{array}{l}\text { No. of } \\
\text { children }\end{array}$ & $\begin{array}{l}\text { Mean age } \pm \text { SD } \\
\text { in years) }\end{array}$ & $\begin{array}{l}\text { Incidence(\%) } \\
\text { of HUS }\end{array}$ & $\begin{array}{l}\text { Mean age of HUS } \\
\text { patients } \pm \text { SD (in } \\
\text { years) }\end{array}$ & Deaths & p value $b$ \\
\hline $\begin{array}{l}\text { Used } \\
\text { Within 3 days } \\
\text { from onset }\end{array}$ & 310 & $7.8 \pm 2.4$ & $57(18.4)$ & $6.8 \pm 2.4$ & 3 & 0.0001 \\
$\begin{array}{l}\text { On or after 4 } \\
\text { days from onset }\end{array}$ & 89 & $8.0 \pm 2.4$ & $23(25.8)$ & $6.7 \pm 2.5$ & 1 & 0.00002 \\
Not used & 19 & $6.7 \pm 2.4$ & $11(57.9)$ & $6.0 \pm 2.3$ & 2 & 0.0139 \\
\hline Total & 329 & $7.7 \pm 2.4$ & $68(20.67)$ & $6.8 \pm 2.4$ & 3 & - \\
\hline
\end{tabular}

a Outbreak-associated cases only; no sporadic infections

$b \quad \chi^{2}$-test compared with patients who were not administered antibiotics.

were given drugs. The incidence of HUS was progressively increased with the day of initiating the antibiotic administration. The incidence of HUS in patients who received antibiotics in the first three days appeared to be significantly lower than in those patients who received antibiotics after 3 days. We further analyzed 329 of the 1271 cases those were bacteriologically or serologically proven to be caused by $E$. coli 0157 during the outbreaks. The ages of patients in this group was also limited to those less than 16 years. The pattern of incidence of HUS among these cases was reminiscent of the pattern seen in all 1271 cases (Table 4). The major reason for the late administration of antibiotics was the delayed visit to physicians or the delayed diagnosis of EHEC infection. Main type (up to 92\%) of antibiotics was fosfomycin (FOM), which blocks initial stage of peptidoglycan synthesis 5). Some previous reports have documented that antibiotics tend to exacerbate the clinical course of the disease; this exacerbation has been related to the intra- and extra-cellular increase in concentration of Stx 6,7). We saw no evidence of exacerbation with antibiotic treatment. The 54 healthy carriers of STEC were reported, including 38 children, of which 45 carried E. coli O157:H7. All these healthy carriers were treated successfully with antibiotics and none experienced any untoward complications 8).

When we analyzed the data on non-HUS and HUS patients associated with EHEC infection in 1997, it was found that culture positivity in HUS group was low and diagnosed by serological criteria, suggesting that the cases of HUS visited late or diagnosed late. The initial day of drug therapy seen in HUS group was late with an average of 4.1 days as compared to 2.6 days for non-HUS group. However, despite of proper 
Table 5. Strategy to prevent the progression of Hemorrhagic Colitis to HUS

- Rapid diagnosis within 3 days of illness.

- Oral administration of antibiotics (FOM).

- Stx-specific adsorbent treatment in the gut.

- Humanized monoclonal anti-Stx antibody is indicative for high risk patients including late diagnosed cases.

treatment with antibiotics, we still had cases with HUS. Additional strategies are needed to prevent the progression of hemorrhagic colitis to HUS.

Synsorb $\mathrm{Pk}$ is synthetic $\mathrm{Pk}$ trisaccharide coupled to Chromosorb $\mathbf{P}$ using spacer sequence of 8-methoxycarbonyloctyl (MCO) devised for a diagnostic or therapeutic agent in diseases caused by Shiga toxin 9). We began exploring the use of Synsorb Pk. The results of in vitro evaluation, we got the evidence of bound Stx1 and Stx2 on Synsorb Pk, and decreased free toxin level in the supernatant. We could also detect Stx on the fecal Synsorb Pk from patients with EHEC 0157:H7 infection. Our experience on the combined usage of FOM and Synsorb $\mathrm{Pk}$ was satisfactory in the sense of fecal toxin level of the patients. In several particular cases, fecal Stx level was very high and the patient was excreting 0157 at early stage of illness. The next day of the treatment, however, the fecal toxin was undetectable and the stool was culture negative indicating that the organism and toxin were eliminated by combined use of antibiotics and Synsorb Pk. These cases successfully recovered.

While we were successful in prevention of progression of EHEC infection to HUS in majority of the cases, we also encountered failures in a few cases. For such rare cases we need more potent treatment such as the use of toxin-oriented specific monoclonal antibody to neutralize the Stx already in the blood stream or in the target tissues. In Table 5, the summary of the strategy to prevent the progression of hemorrhagic colitis to HUS is shown.

\section{REFERENCES}

1. Su, C. and Brandt, L.J. (1995): Escherichia coli O157:H7 infection in humans. Ann Intern Med 123 : 698-714.

2. Cimolai, N., et al. (1990): Risk factors for the progression of Escherichia coli O157:H7 enteritis to hemolytic-uremic syndrome. J Pediatr $116: 589-592$.

3. Watanabe, H., et al. (1996): Outbreaks of enterohaemorrhagic Escherichia coli O157:H7 infection by two different genotype-strains in Japan, 1996. Lancet $348: 831-832$.

4. Izumiya, H., et al. (1997): Molecular typing of enterohemorrhagic Escherichia 
coli $\mathrm{O} 157: \mathrm{H7}$ isolates in Japan using pulsed-field gel electrophoresis and random amplified polymorphic DNA analysis. J. Clin. Microbiol.

5. Kahan, F.M., et al. (1974): The mechanism of action of fosfomycin (phosphonomycin). Ann New York Acad Sci 235 :364-386.

6. Karch, H., Strockbine, N.A. and O'Brien, A.D. (1986): Growth of Escherichia coli in the presence of trimethoprim-sulfamethoxazole facilitates detection of Shiga-like toxin producing strains by colony blot assay. FEMS Microbiol Lett. $35: 141-145$.

7. Walterspiel, J.N., et al. (1992): Cleary TG. Effect of subinhibitory concentrations of antibiotics on extracellular Shiga-like toxin I. Infection, $20: 25-29$.

8. Takeda, T., et al. (1998): Early use of fosfomycin for STEC 0157 infection reduces the risk of hemolytic uremic syndrome. Kaper, J., and O'Brien, A., eds. E. coli O157:H7. ASM Press, 385-387.

9. Armstrong, G.D., et al. (1995): A phase I study of chemically synthesized verotoxin (shiga-like toxin) Pk-trisaccharide receptors attached to Chromosorb for preventing hemolytic uremic syndrome. J. Infect. Dis. 171 : 1042-1045. 